\title{
Structural and Kinematic Parameters of the Two Halo Components of the Milky Way: Preliminary Results
}

\author{
Daniela Carollo $^{* a}$, Timothy C. Beers ${ }^{c}$, Masashi Chiba ${ }^{d}$, John E. Norris ${ }^{e}$, Young Sun \\ Lee $^{c}$, Kenneth C. Freeman ${ }^{e}$ \\ ${ }^{a}$ Research School of Astronomy \& Astrophysics, Australian National University \& Mount Stromlo \\ Observatory, and INAF-Osservatorio Astronomico di Torino - Italy \\ ${ }^{c}$ JINA: Joint Institute for Nuclear Astrophysics, Dept. of Physics \& Astronomy, Michigan State \\ University, USA \\ ${ }^{d}$ Astronomical Institute, Tohoku University, Sendai, Japan \\ ${ }^{e}$ Research School of Astronomy \& Astrophisics, Australian National University \& Mount Stromlo \\ Observatory, ACT, Australia \\ carollo@mso.anu.edu.au,beers@pa.msu.edu,chiba@astr.tohoku.ac.jp, \\ jen@mso.anu.edu.au, lee@pa.msu.edu,kcf@mso.anu.edu.au
}

\begin{abstract}
The structure of the halo of the Milky Way has recently been shown to be clearly divisible into two overlapping stellar components, the inner and the outer halo. The first structure dominates at Galactocentric distances $\mathrm{R}<10-15 \mathrm{kpc}$, exhibits highly eccentric orbits, has a slightly prograde rotation, and comprises stars with a peak in their metallicity distribution function (MDF) around $[\mathrm{Fe} / \mathrm{H}]=-1.6$. The outer-halo component dominates at $\mathrm{R}>15-20 \mathrm{kpc}$, exhibits a much more uniform distribution of orbital eccentricities, has a clear (and statistically significant) net retrograde rotation, and comprises stars with a peak in their MDF a factor of three lower than the inner halo $([\mathrm{Fe} / \mathrm{H}]=-2.2)$. Such properties indicate that one might associate two distinct modes of formation, and timescales, for the assembly of these two structures. The dataset upon which these claims are based has recently been increased by $50 \%$ with the addition of new stars from SDSS/SEGUE. We present the first results of a Maximum Likelihood technique that provides estimates for the structural parameters of the inner- and outer-halo components, including estimates of the fraction of stars within each population as a function of distance. Such numbers are critical for refining ongoing and future searches for the most metal-poor stars in the Galaxy
\end{abstract}

10th Symposium on Nuclei in the Cosmos

July 27 - August 12008

Mackinac Island, Michigan, USA

\footnotetext{
*Speaker.
} 


\section{Introduction}

Using the sample of calibration stars of the fifth data release (DR-5) of the Sloan Digital Sky Survey (SDSS), we obtained signatures of the presence of, at least, two stellar components in the Galactic halo [1]. We are now working to quantify the structural and kinematic parameters for these two components, as well as for all the other recognized structures of our Galaxy. The analysis uses a Maximum Likelihood (ML) technique, which provides the velocity ellipsoids, and the fraction of stars for each component as a function of $\mathrm{Z}_{\max }$ (the maximum distance above or below the Galactic plane achieved by a star during its orbit). This analysis uses the the sixth data release of SDSS/SEGUE (DR-6; [2]), which comprises more than 35000 calibration stars, but the final results will be obtained with the SDSS/SEGUE DR-7 sample, which increase the sample to around 40000 stars (see also Beers et al. 2008, this volume).

\section{Decoupling the Inner/Outer Halo}

Maximum Likelihood analysis is a well known general technique of statistical estimation. In our case the likelihood function comprises four Gaussian functions, which represent the probability distribution of the Galactocentric rotational velocity of the thick disk, the metal-weak thick disk (MWTD), the inner halo, and the outer halo. These components are strongly overlapped in their spatial and metallicity distributions, thus it is not easy to disentangle each structure. In our ML approach, we first considered the extremes of the metallicity range, then derived the trend of the Galactocentric rotational velocity (rotational velocity measured in the Galactocentric cylindrical reference frame, $\left.\mathrm{V}_{\phi}\right)$, and its dispersion $\left(\sigma_{V_{\phi}}\right)$ as a function of $\mathrm{Z}_{\max }$. In these regions of metallicity, we sample stars belonging mostly to the outer halo in the first case, and to the thick disk in the second case. Once we have derived the value of $\left\langle\mathrm{V}_{\phi}\right\rangle$ and $\sigma_{V_{\phi}}$ for the outer halo, we can use them as input parameters in the ML analysis in the low-metallicity range $([\mathrm{Fe} / \mathrm{H}]<-2)$, and fit the two halo components (the thick disk and MWTD are not present in this region of $[\mathrm{Fe} / \mathrm{H}]$ ). This fit provides the value of $\left\langle\mathrm{V}_{\phi}\right\rangle$ and $\sigma_{V_{\phi}}$ for the inner-halo component. Then we can extend our $\mathrm{ML}$ analysis to the full range of metallicity, and fit for three components, the thick disk, inner halo, and outer halo. Finally, we explore the data in the intermediate metallicity range, and fit for three components, the inner halo, metal-weak thick disk, and thick disk. The full analysis procedure is to be included in a paper in preparation.

\section{Exploring the Data at the Extremes of the Metallicity Range}

We have considered the range of metallicity, $[\mathrm{Fe} / \mathrm{H}]<-2$, in which the sample is dominated by outer-halo stars, and the range, $-0.8<[\mathrm{Fe} / \mathrm{H}]<-0.6$, where only the thick disk is present. Figure 1 shows the trend of $\left\langle\mathrm{V}_{\phi}\right\rangle$ and $\sigma_{V_{\phi}}$ in these ranges of [Fe/H]. It is worth noting, in the bottom left panel, that the rotational velocity exhibits an abrupt change between $5 \mathrm{kpc}<\mathrm{Z}_{\max }<$ $10 \mathrm{kpc}$, and then remains constant for $\mathrm{Z}_{\max }$ greater than $10 \mathrm{kpc}$, at a value of $\left\langle\mathrm{V}_{\phi}\right\rangle=-85 \mathrm{kms}^{-1}$, which is even more retrograde than the value obtained for the outer halo previously [1]. We derive a value for the dispersion of the outer halo of the order of $\sigma_{V_{\phi}}=145 \mathrm{kms}^{-1}$. The high metallicity range adopted for the thick-disk component provides $\left\langle\mathrm{V}_{\phi}\right\rangle=170 \mathrm{kms}^{-1}$, and $\sigma_{V_{\phi}}=60 \mathrm{kms}^{-1}$ for the rotational velocity, and the velocity dispersion, respectively. 

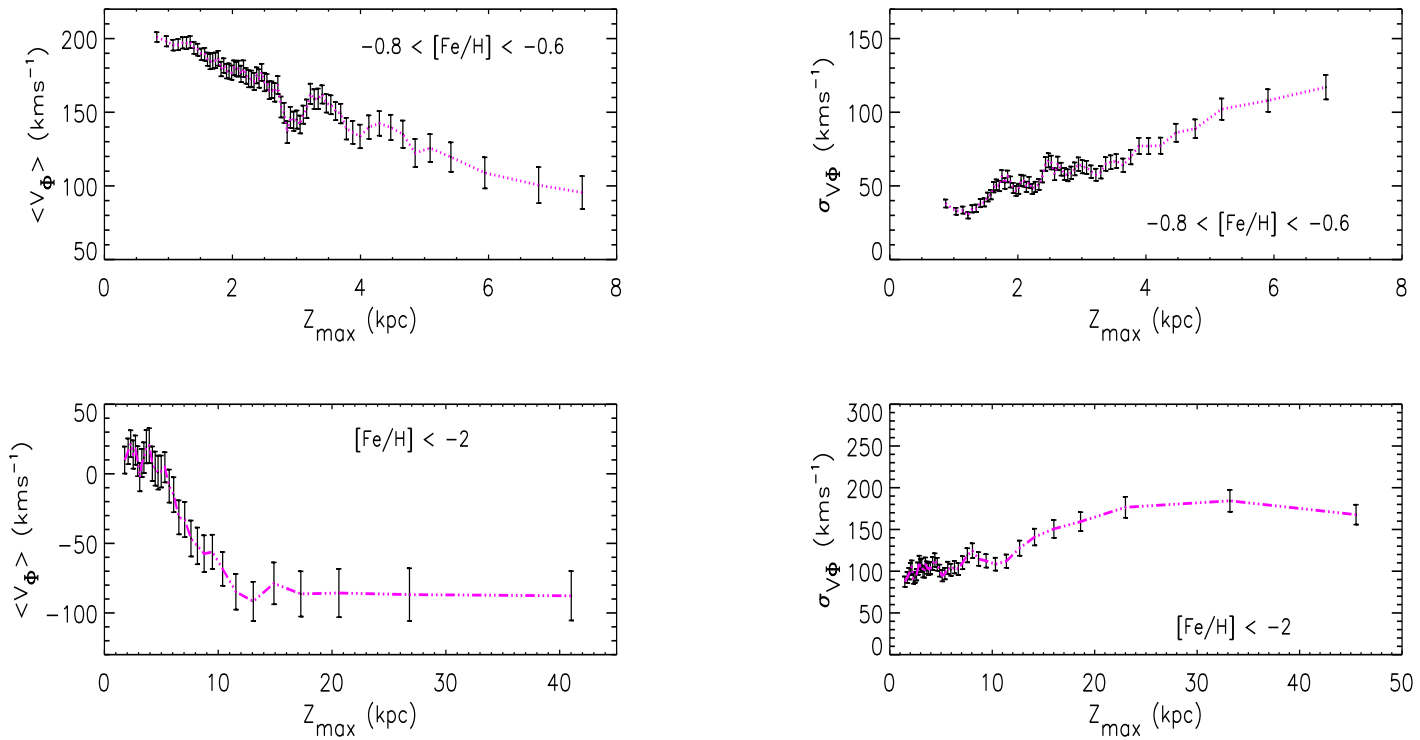

Figure 1: Left panel: Mean galactocentric rotational velocity as a function of $Z_{\max }$ for the thick disk (top), and the outer halo (bottom). Right panel: velocity dispersion for the same components as a function of $Z_{\max }$. The values of $\mathrm{V}_{\phi}$ and $\sigma_{V_{\phi}}$ are evaluated by passing a box of 100 stars with an overlap of 50 stars.

\section{Low Metallicity Range: Results of the ML Analysis}

At low metallicity only two components are present, the inner and outer halo, and we perform a fit of this subset of stars using a mixture of two Gaussian distributions for $\left\langle\mathrm{V}_{\phi}\right\rangle$. The input parameters are $\left\langle\mathrm{V}_{\phi}>\right.$ and $\sigma_{V_{\phi}}$ of the outer halo. The output is the rotational velocity and dispersion for the inner halo. Figure 2 shows the cumulative distribution of $\mathrm{V}_{\phi}$ for increasing values of $\mathrm{Z}_{\max }$. In this Figure the histograms denote the distribution of the subset of stars in each range of $Z_{\max }$, while the smooth curves, green (inner halo) and red (outer halo), represent the results of the ML analysis for reproducing the $\mathrm{V}_{\phi}$ distribution. The blue smooth curve is the mixture of the two Gaussian components. It can be noted that the fit reproduces very well the observed distribution of the data, and as expected, the fraction of stars of the outer halo increases at higher values of $\mathrm{Z}_{\text {max }}$, while the inner halo has the opposite trend. In each panel is also indicated the fraction of stars for each component provided by the fit. The value of the rotational velocity and the dispersion obtained for the inner halo are $\left\langle\mathrm{V}_{\phi}\right\rangle=20 \mathrm{kms}^{-1}$ and $\sigma_{V_{\phi}}=100 \mathrm{kms}^{-1}$, respectively. It is worth noting that the value of the dispersion obtained is in agreement with that of [3].

\section{The Full Metallicity Range ML Analysis}

The low $[\mathrm{Fe} / \mathrm{H}]$ range $\mathrm{ML}$ analysis has provided two parameters for the inner halo, $\left\langle\mathrm{V}_{\phi}\right\rangle$ and $\sigma_{V_{\phi}}$, which can now be used to fit the data in the case of three components: the thick disk, inner halo, and outer halo. The likelihood function now includes three Gaussian distributions, and the output parameters are the fractions of stars for each component, $\mathrm{F}_{T D}, \mathrm{~F}_{I N}$, and $\mathrm{F}_{O U T}$. The right 

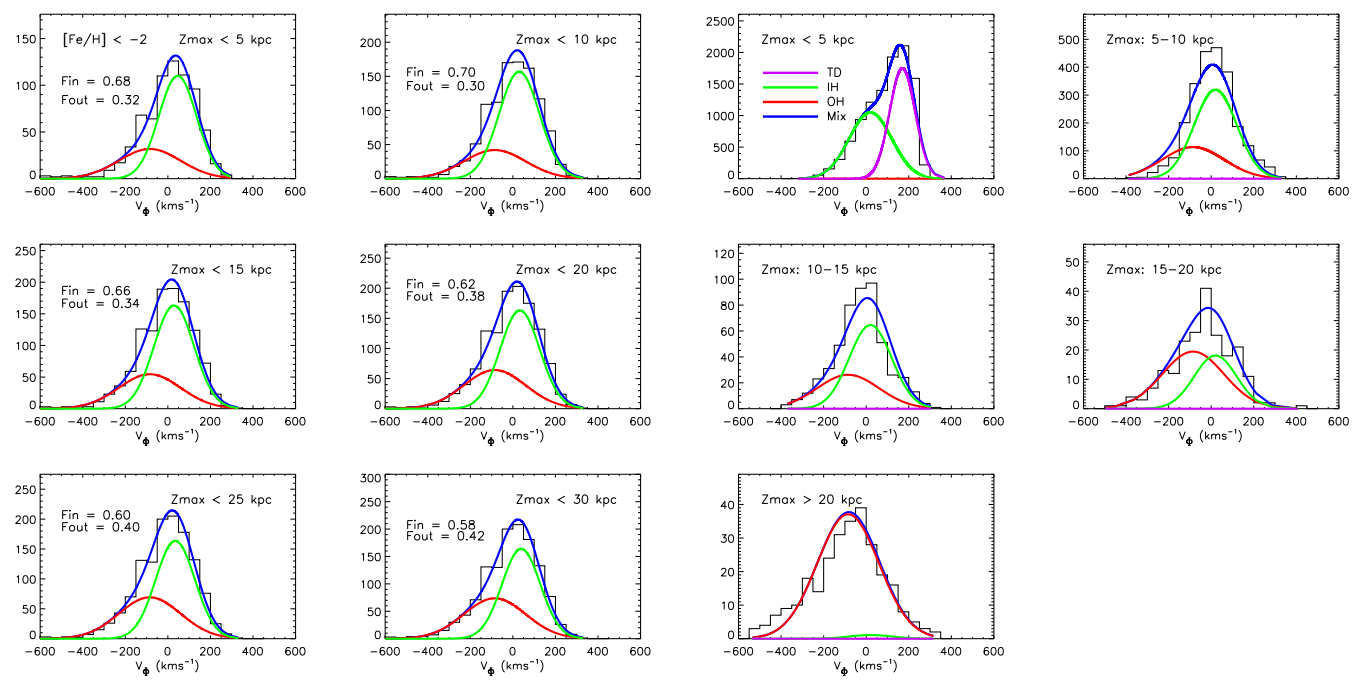

Figure 2: Left panels: Cumulative distribution for the low $[\mathrm{Fe} / \mathrm{H}]$ range. Right panels: Differential distribution for the full $[\mathrm{Fe} / \mathrm{H}]$ range. See text for the description.

panel of Figure 2 shows the differential distribution, i.e., the fit obtained for different ranges of $Z_{\max }$, as indicated in each panel. The differential distribution differs from the cumulative one because it selects sub-samples of stars, at ranges of distance that increase progressively, while the cumulative distribution adds stars for each value of increasing $\mathrm{Z}_{\max }$. Thus, the differential distribution is able to recognize the change in the dominance of the overlapped structures. In the Figure, the meaning of the histograms and the smooth curves remains the same as in the low metallicity case, with the exception that now there is a third component, the thick disk, which is drawn as a purple curve. Notice that, at $Z_{\max }<5 \mathrm{kpc}$, just two components are present, the thick disk and the inner halo. The outer halo starts to appear above $5 \mathrm{kpc}$, and it becomes more dominant as the distance increases. At the same time, the fraction of stars of the inner halo decreases. At $15<\mathrm{Z}_{\max }<20$, the inner and outer halo are comparable, and finally, when $Z_{\max }>20 \mathrm{kpc}$, the outer halo is completely dominant. This second ML analysis provides the fraction of stars for the three stellar population as a function of $\mathrm{Z}_{\text {max }}$ (Carollo et al., in preparation).

\section{Decoupling the Thick Disk and MWTD}

The intermediate metallicity range $(-1.6<[\mathrm{Fe} / \mathrm{H}]<-0.8)$ is suitable to explore and extract information on the metal weak thick disk. The MWTD could be thought as the low metallicity tail extension of the thick disk, or as an independent structural component of the Galaxy. We applied the ML analysis to the sub-sample of stars in this range of $[\mathrm{Fe} / \mathrm{H}]$ assuming the MWTD is an independent component. In this case, the likelihood function comprises three components, the inner halo, the metal weak thick disk, and the thick disk. The result is shown in Figure 3, where the orange smooth Gaussian denotes the MWTD. The derived fraction of stars for the inner halo, MWTD, and thick disk is $F_{I N}=0.41, F_{T D}=0.38, F_{M W T D}=0.21$, respectively, as also reported in Figure 3. The ML analysis provides also the rotational velocity and its dispersion for the MWTD component, which are $\left\langle\mathrm{V}_{\phi}\right\rangle=90 \mathrm{kms}^{-1}$ and $\sigma_{V_{\phi}}=70 \mathrm{kms}^{-1}$, respectively. 


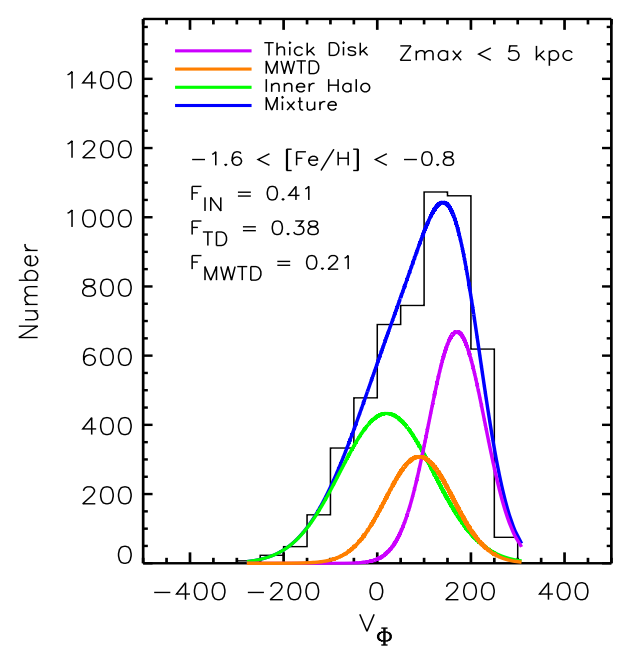

Figure 3: Cumulative distribution at $\mathrm{Z}_{\max } 5 \mathrm{kpc}$ in case of the intermediate metallicity range.

\section{Work in Progress}

We have presented the preliminary results of a maximum likelihood analysis applied to the calibration stars of SDSS/SEGUE DR-6. We obtained the first component of the velocity ellipsoids (in the rotational direction) for the thick disk, metal-weak thick disk, inner halo, and outer halo. The complete analysis will use the maximum likelihood technique applied to the remaining velocity components $\left(\mathrm{V}_{R}, \mathrm{~V}_{Z}\right)$, the derivation of the spatial distribution in the Z-R plane and the evaluation of the scale length and scale height for each component. Also, we will explore a possible correlation between the kinematics and other chemical tracers, such as $\alpha / \mathrm{Fe}]$ and $[\mathrm{C} /[\mathrm{Fe}]$. A preliminary result related to the alpha elements is presented by Lee et al. (this volume).

\section{References}

[1] Carollo, D., Beers, T.C., Lee, Y.S., Chiba, M., Norris, J.E., et al. 2007, Two Stellar Components in the Halo of the Milky Way, Nature, 450, 1020

[2] Adelman-McCarthy, J.K, Agüeros, M.A., Allah, S.S, Allende Prieto, C., Anderson, K.S.J., et al. 2008, The Sixth Data Release of the Sloan Digital Sky Survey, ApJS, 175, 297

[3] Chiba M., \& Beers T.C., 2000, Kinematics of metal-poor stars in the Galaxy. III. Formation of the stellar halo and thick disk as revealed from a large sample of non-kinematically selected stars, AJ, 119, 2843 